\title{
Surgical Approaches and Postoperative Complcations of Parapharyngeal Space Tumours
}

\author{
Anna Ivanova*, Juris Tars*, Valerija Groma** \\ * Department of Head and Neck Surgery, Riga East University Hospital, Oncology Center of Latvia \\ ** Institute of Anatomy and Anthropology, Riga Stradins University, Latvia
}

\section{Summary}

Introduction. Parapharyngeal space (PPS) tumors are rare neoplasms that are greatly varying both clinically and morphologically. Complete tumor removal from the PPS requires an integrated approach to resection of nervous and vascular trunks and hemorrhage control.

Aim of the Study. This study aimed evaluation of surgical interventions applied for PPS neoplasms along with revision of postoperative complications noticed conducting these surgeries.

Material and Methods. 32 PPS tumors removed during surgeries as well as associated biopsies performed at Riga East University Hospital Oncology Center of Latvia at the Department of Head and Neck Surgery from 01.01.2001 till 31.12.2006 were included in this study.

Results. The largest number of patients presented with benign salivary gland tumors $22(68.7 \%)$, followed by malignant salivary gland neoplasms 5 (18.5\%), neurogenic tumors 4 (12.5\%), and miscellaneous tumors $1(0.3 \%)$. Postoperative complications for malignant salivary gland surgeries occurred at the highest rate $(42 \%)$, whereas, for benign - at the lowest $(5 \%)$. Transcervical submandibular, transparotid, and transoral approach was used in 56,25 , and $1 \%$ of surgeries, accordingly, whereas, mandibulotomy applied in 17\%. "First bite syndrome" was noticed in 30\% of the cases, cranial nerve palsies - in $20 \%$, temporary, permanent facial nerve and accessorial nerve injury in 4,1 and $15 \%$ of surgical interventions, accordingly. Vascular trunks damage occurred at $9 \%$, and, mostly, for poststyloid lesions.

Conclusions. Heterogeneous PPS tumors show a wide spectrum of postoperative complications and require a selective approach for optimization of surgical intervention and treatment strategy.

Key words: parapharyngeal space tumors, transcervical, transparotid and transoral approach, postoperative complications

\section{INTRODUCTION}

Oncologic processes developing within the parapharyngeal space (PPS) lead to appearance of neoplasms subdivided into three categories: salivary gland tumors, neurogenic tumors and miscellaneous tumors. Besides salivary gland primary tumors a direct extension from the deep lobe of parotid gland occurs as well as metastases from elsewhere $(9,11)$. Clinical manifestations that may direct clinician to a PPS tumor are presented with upper neck or oropharyngeal mass, unilateral Eustachian tube dysfunction, dysphagia, obstructive sleep apnea, cranial nerve deficits, Horner syndrome, pain and trismus (1). Often, however, these tumors even when large present with very little or no symptoms. It may be an incidental finding on an imaging study done for some other reason.

Parapharyngeal space tumors are rare challenging lesions that require a proper understanding of anatomic complexity. The variety of pathologic entities demands a clear understanding of management strategies (5). The goal of parapharyngeal surgery is achievement of complete tumor removal with preservation of integrity of surrounding nerves and vessels and controlling any hemorrhage performed after adequate tumor visualization. Many surgical approaches have been reported in the literature $(1,4,5,8,9)$ but in overall, transcervical and transparotid approach are the main two. They have also been used with mandibulotomy to increase exposure during surgery $(1,4,7)$.

Analysis of surgical interventions performed by Latvian specialists and applied due to removal of heterogeneous tumors developed within the PPS has not been conducted until now. Summarization of data on the surgeries conducted at Riga East University Hospital Oncology Center of Latvia at the Department of Head and Neck Surgery between 01.01.2001 and 31.12.2006, and revision of postoperative complications was accompanied by analysis of head and neck neoplasms affecting the Latvian population. In this study, we analyzed the relative frequency and distribution of the various types of benign and malignant salivary gland tumors, neurogenic tumors, and miscellaneous neoplasms occurring in the PPS.

\section{AIM OF THE STUDY}

In this study we paid a special attention to analysis of surgical interventions conducted using transcervical, transparotid and transoral approach for neoplasm removal and revise complications arising in postoperative period in various types of PPS tumors.

\section{MATERIALS AND METHODS}

32 PPS tumors removed during surgeries as well as associated biopsies performed at Riga East University 
Hospital Oncology Center of Latvia at the Department of Head and Neck Surgery from 01.01.2001 till 31.12.2006 were included in this study. Patients were divided into four groups according to the type of PPS pathology: group A $(n=22)$ - benign salivary gland tumors; group $B$ $(n=5)$ - malignant salivary gland tumors; group $C(n=4)-$ neurogenic tumors; group D $(n=1)$ - miscellaneous tumors. Patients were followed-up for three years in order to evaluate the impact of surgical complications on later outcomes. Clinical, laboratory and instrumental data were obtained from medical records. Results were summarized using descriptive statistics methods, and the data on types of PPS tumors, rates and types of postoperative complications were systematized using Microsoft Excel data processing program.

\section{RESULTS}

Group A constituted a majority of tumors $68.7 \% \quad(22$ cases) presented with benign salivary gland neoplasms (Fig. 1). From them, eighty percent were pleomorphic adenomas. In group B constituting $18.5 \%$ (5 cases) mucoepidermoid carcinoma was the most common malignant tumor followed by carcinoma ex pleomorphic adenoma. Parapharyngeal neurogenic tumors included in the group C constituted $12.5 \%$ (4 cases). These commonly presented with a poststyloid mass, and were subdivided into paragangliomas, carotid body tumors, and vagal paragangliomas or schwannomas. Miscellaneous tumors were rare and heterogeneous neoplasms - 0.3\% (1 case). Usually these included lymphomas, hemangiomas, teratomas, lipomas and arteriovenous malformations. The case appeared under the scope of this study was lymphoproliferative disease. Several surgical approaches to the PPS based on severity of the neoplasm confirmed by histopathologic examination and the exact extend of tumor were used. The transoral approach was used in $1 \%$ of the cases for removal of small, benign neoplasms that originate in the prestyloid PPS and present with an oropharyngeal mass. Limitations of this approach were the restricted access, inability to visualize great vessels, increased risk of facial nerve injury and tumor rupture. The transoral approach may be combined with an external approach to fix lesions with significant oropharyngeal component.

Transcervical - submandibular approach was used in $56 \%$ of surgeries. The division of the stylomandibular ligament and retraction of the mandible was used to enlarge the access. Sometimes some pressure through the mouth appeared to be necessary to expel the tumor. Transparotid - cervical approach was used in 25\% of the cases. This approach combined a parotidectomy approach and visualization of the main trunk of the facial nerve and its lower or all branches with a transcervical approach. This method was applied when tumor was attached to or originated from the deep lobe of the parotid gland.

Extended approaches with the mandibulotomy, which gave an excellent exposure to the PPS but was associated with certain morbidity and, therefore, was applied for extensive vascular tumors or recurrent pleomorphic adenoma with multiple large nodules was used in 17\% of cases. Various locations for osteotomy have been used including mandibular body, angle, ramus, and parasymphyseal. For lesions localized at the base of the skull an infratemporal fossa approach was needed in $1 \%$.

A rate of complications varied among the groups studied (Fig. 2). The highest rate constituting 45\% was demonstrated for B group patients, whereas, the lowest $5 \%$ - for A group patients. The spectra and rates of greatly varying postoperative complications occurring in surgical interventions in PPS are summarized at the Figure 3. A common complication in $30 \%$ of the cases was so-called "first bite syndrome". Other complications that occurred much less frequently included temporarily facial nerve weakness. The temporary injury of the facial nerve from traction affection and permanent due to inadvertently sacrificing or affected manipulation was appearing in 4 and $1 \%$ of surgical interventions, accordingly.

Cranial nerve palsies were demonstrated in $20 \%$ of the cases resulted from removal of poststyloid PPS lesions. Injury of the spinal accessories nerve occurred in 15\% of the cases and manifested with weakness of the trapezius muscle, winging of the scapula, and adhesive capsulitis. Tracheostomy was required for airway protection in $0.5 \%$ of the cases when multiple cranial nerve deficits appeared from resection. Palatal weakness, vocal cord paralysis and fistula were fixed very rarely $(0.5 \%)$. Cerebrospinal fluid leaks was detected after removal of tumors with jugular foramen or intracranial extension in $1 \%$ of the cases. Fascial, adipose or muscular material was used to reinforce the closure.

Vascular complications were commonly associated with removal of neurogenic or vascular lesions. The prevalence of intraoperative vascular injury and of perioperative stroke has been detected at $9 \%$ for poststyloid lesions. Avoidance of undue traction on the carotid artery was used for reduction of morbidity, sometimes this pathway resulted in intimal tears. Primary repair or vein grafting was performed for all vessel lacerations.

Injury to the lingual and hypoglossal nerves developed from the transcervical approach when the submandibular triangle was entered was noticed $(5 \%)$ in addition to the postoperative complications discussed above. Isolated hypoglossal nerve damage did not usually significantly impair swallowing or speech function.

The vagus nerve was the most commonly affected nerve. This injury presented with a vocal cord paralysis, and if injury occurred above the level of the nodose ganglion, laryngeal sensation was also affected.

Complications of mandibulotomy included infection, temporomandibular joint dysfunction, nonunion, plate extrusion, and tooth loss in 5\% of the cases. When the osteotomy site was through dental sockets rather than between them, tooth loss was more common.

The recurrence rate of benign PPS neoplasms following surgical extirpation constituted $9 \%$. 


\section{DISCUSSION}

Primary parapharyngeal tumors are rare and these are located in a complex anatomical region. Clinical presentation of these tumors can be subtle. Parapharyngeal salivary gland tumors are the most common form of PPS neoplasms, and occur in the prestyloids compartment. Surgical resection is the mainstay of treatment. Transcervial-transparotid approach with or without mandibulotomy is preferred by most surgeons. The transoral is not the approach of choice for most lesions of the PPS (13).

The "first bite syndrome" is the most common complication arising from the damage of the sympathetic innervation of the parotid gland. The myoepithelial cells of the parotid gland may cause supramaximal contraction during the first bite of a meal and give the patient excruciating pain. It has been shown very recently in the paper published by Costa T.P. et al. (2012) that botulin toxin type A has been suggested as a treatment (6).

According to the literature, the risk of postoperative cranial nerve deficits ranges from $11-57 \%(7,10,11)$, with higher frequencies observed in studies of patients with proportionately greater numbers of malignancies or neurogenic lesions. An isolated nerve injury is usually well tolerated in an otherwise healthy patient. Intraoperative transection of the facial nerve is best managed by performing nerve grafting at the time of surgery. Eye protection is required in the postoperative period - insertion of a gold weight can be performed at surgery or postoperatively.

An isolated unilateral vagal injury is usually well tolerated in the otherwise healthy patient, but a high vagal injury or a vagal injury combined with injury to cranial nerve $(\mathrm{CN})$ IX - n.glossopharyngeus and CN XII n.hypoglossus, may result in significant problems with swallowing and aspiration. Patients should be carefully evaluated prior to the institution of oral feedings, and an alternate method of feeding (nasogastric tube, gastric tube) should be instituted if necessary $(6,7)$.

Elderly patients and/or patients with multiple $\mathrm{CN}$ deficits are expected to have greater difficulty with swallowing, and medialization as well as cricopharyngeal myotomy should be performed concomitant with resection. The advantage to delaying vocal cord medialization procedures is that patients often compensate, and the extent of medialization required can be better assessed after this has occurred (3).

If swallowing rehabilitation is prolonged or unsuccessful, gastrostomy tube placement may be necessary. Patients with difficulty handling oral secretions may require tracheostomy for airway protection.

Injury to the spinal accessory nerve can be managed postoperatively by an active range of motion physical therapy program. According to Khafif A. et al. (2005), when recognized intraoperatively, transection of $\mathrm{CN}$ $\mathrm{XI}$ is best managed by nerve grafting to achieve some recovery of function (10).

Horner syndrome may result from injury to the cervical sympathetic chain. The resulting anhydrosis is managed symptomatically.
Since primary parapharyngeal tumors are exceedingly rare, only very few large cohort studies are available. Shahab R. et al. (2005), reviewed 114 parapharyngeal tumors treated over 27 years of experience, whereas, the second study revising large clinical cohort is published recently by Sang J. et al., 2011 (12). According to the literature (2), paragangliomas recur in approximately $5 \%$ of cases, and, since $10 \%$ are multicentric, the risk of developing a second tumor remains. Patients with a familial paraganglioma syndrome have a $35 \%$ risk of multicentricity. In addition, patients with paragangliomas who are being treated nonoperatively must be alerted to the risk of malignant degeneration, which constitutes approximately $10 \%$ and is usually associated with rapid growth.

Malignant tumors of the PPS have a much higher rate of recurrence - $25-77 \%$, depending on histology, extent of resection, and duration of follow-up (9). Postoperative radiation therapy for PPS malignancies is recommended to prevent recurrences; however, because of the relative scarcity of these lesions, no large series are available to demonstrate a survival benefit (11).

The 5-year and 10 year survival for benign PPS tumor is $100 \%$. For malignancies the 5 year survival was $93 \%$, but fall to $57 \%$ at 10 years. This study showed that a patient is highly unlikely to die of a benign PPS tumor, therefore careful consideration of surgical treatment and discussion with the patient are crucial. While surgery is the mainstay of the treatment for parapharyngeal tumor, radiation therapy should be considered in elderly patients with paragangliomas. According to the literature (11), isolated asymptomatic parapharyngeal schwannomas in elderly patients with no nerve deficit should probably be suspected.

Mandibulotomy can be recommended for vascular tumors extending into the superior PPS, solid tumors that are confined to the superior aspect of the PPS and malignant tumors invading the skull base $(8,13,14)$.

Preoperative embolization of vascular lesions may decrease intraoperative blood loss and may facilitate dissection of tumors at the skull base by causing retraction. Embolization is recommended for vascular lesions greater than $3 \mathrm{~cm}$ in which obvious feeding vessels can be identified on angiography (10).

The use of embolization of paragangliomas is more controversial. Glomus vagale tumors rarely have a single blood supply, and resection is not associated with excessive blood loss. Embolization of a carotid body tumor may cause an inflammatory response that may obscure the subadventitial plane in which the tumor is dissected, increasing the risk of inadvertent carotid injury during resection. Embolization carries a risk of possible disruption of the blood supply to cranial nerves. If embolization is performed, it is performed within 24 hours prior to resection. Costa T.P. et al. (2012) publication showed that factors as age and multiplicity may also come into the equation when neurologic injury is to be expected. For some patients observation may be the best option, even though the tumor might be technically respectable (6). 
It is important to avoid bilateral cranial nerve paralysis in patients with multiple lesions or who have a family history, especially the vagal and hypoglossal nerves. The arguments in favor of observation are that paragangliomas grow very little per year in the order of $1 \mathrm{~mm}$, and they are almost always benign tumors, therefore, morbidity and mortality is low if tumor left untreated.

\section{CONCLUSIONS}

The present study showed that neoplasms resected from the PPS are benign and malignant salivary gland tumors, neurogenic and miscellaneous tumors. The transcervical, transparotid and transoral approach were used for removal of neoplasms. The practical evidences obtained during surgeries and patients' follow-up as well as revision of postoperative complications of heterogeneous PPS tumors is useful for selection of surgical strategy and management of clinical course of tumor and outcome of disease when factors such as anatomical regional complexity, severity and recurrence of neoplasm, age, and other parameters should be taken into consideration.

\section{Conflict of interest: None}

\section{REFERENCES}

1. Ahmad F, Waqar-uddin, Khan MY, Khawar A, Bangush W, Aslam J. Management of parapharyngeal space tumours // J Coll Physicians Surg Pak, 2006; 16:7-10

2. Boedeker CC, Ridder GJ, Schipper J. Paragangliomas of the head and neck: diagnosis and treatment // Fam Cancer, 2005; 4:55-59

3. Bozza F, Vigili MG, Ruscito P, Marzetti A, Marzetti F. Surgical management of parapharyngeal space tumours: results of 10-year follow-up // Acta Otorhinolaryngol Ital, 2009; 2:10-15

4. Bradley PJ, Bradley PT, Olsen KD. Update on the management of parapharyngeal tumours // Laryngoscope, 1994; 5:1-28

5. Cohen SM, Burkey BB, Netterville JL. Surgical management of parapharyngeal space masses // Head Neck, 2005; 27:669-675

6. Costa TP, de Araujo CE, Filipe J, Pereira AM. Firstbite syndrome in oncologic patients // Eur Arch Otorhinolaryngol, 2012; 1:265-273

7. Dimitrijevic MV, Jesic SD, Mikic AA, Arsovic NA, Tomanovic NR. Parapharyngeal space tumors: 61 case reviews // Laryngoscope, 2010; 8:1557-1562

8. Flood TR, Hislop WS. A modified surgical approach for parapharyngeal space tumours: use of the inverted 'L' osteotomy // Br J Oral Maxillofac Surg, $1991 ; 4: 82-86$

9. Hughes KV, Olsen KD, McCaffrey TV. Parapharyngeal space neoplasms // Head Neck, 1995; 17:124-130

10. Khafif A, Segev Y, Kaplan DM, Gil Z, Fliss DM. Surgical management of parapharyngeal space tumors: a 10-year review // Otolaryngol Head Neck Surg, 2005; 132:401-406
11. Olsen KD. Tumors and surgery of the parapharyngeal space // Laryngoscope, 1994; 104:1-28

12. Sang J, Lou W, Zhang Y. Diagnosis and surgical approach of parapharyngeal space neoplasms // Oral Maxillofac Surg, 2011; 4:211-216

13. Shahab R, Heliwell T, Jones AS. How we do it: A series of 114 primary pharyngeal space neoplasms // Clinical Otolaryngology, 2005; 30:364-367

14. Teng MS, Gender EM, Buchbinder D, Urken ML. Subcutaneous mandibulotomy: a new surgical access for large tumors of the parapharyngeal space // Laryngoscope, 2003;1 13:1893-1897

\section{Address:}

Anna Ivanova

Riga East University Hospital,

Oncology Center of Latvia,

Department of Head and Neck Surgery

2, Hipokrata str.,

Riga, LV 1038, Latvia

\section{Parapharyngeal tumors}

ar.A salivary gl.begin Tu $\quad$ gr.B salivary gl.malignant Tu - gr.C neurogenic Tu $\quad$ gr.D miscellaneous Tu

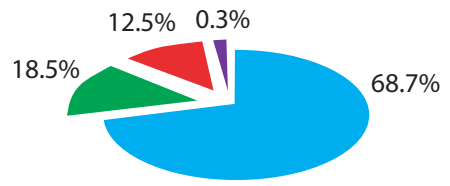

Fig. 1. Spectra and rates of PPS tumors.

\section{Rate of complications}

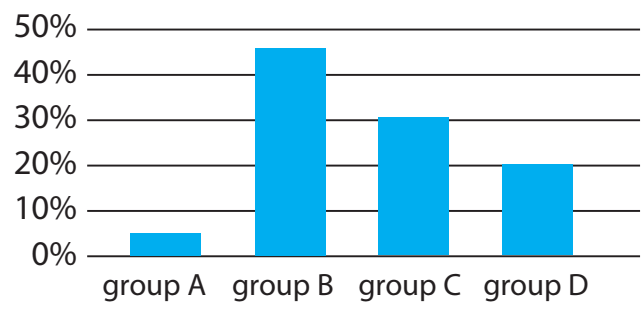

Fig. 2. Rates of postoperative complications occurring after surgical removal of PPS tumors.

\section{Complications of PPS surgery}

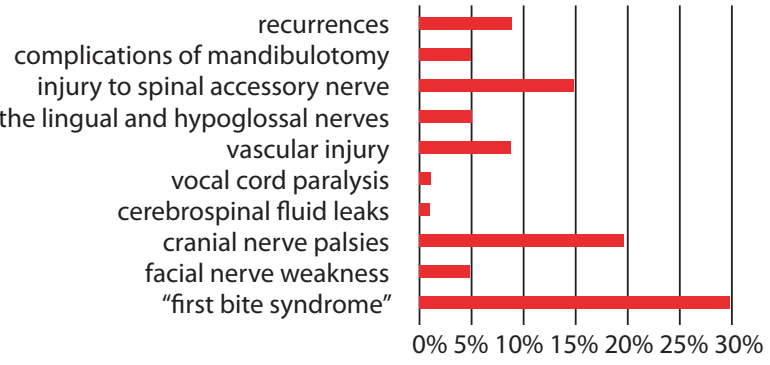

Fig. 3. Spectra of postoperative complications in PPS surgery. 